\title{
On the maximum energy of protons in the hotspots of AGN jets
}

\author{
Anabella T. Araudo ${ }^{1,2, *}$, Anthony R. Bell ${ }^{2}$, James Matthews ${ }^{3}$, and Katherine Blundell ${ }^{3}$ \\ ${ }^{1}$ Astronomical Institute, Czech Academy of Sciences, Bočni II 1401, CZ-141 00 Prague, Czech Republic \\ ${ }^{2}$ ELI Beamlines, Za Radnicí 835, 25241 Dolní Břežany, Czech Republic \\ ${ }^{3}$ Clarendon Laboratory, University of Oxford, Parks Road, Oxford OX1 3PU, UK \\ ${ }^{4}$ Astrophysics, University of Oxford, Keble Road, Oxford, OX1 3RH, UK
}

\begin{abstract}
We study particle acceleration and magnetic field amplification in the termination shocks (hotspots) of radiogalaxy jets. The cut-off of the synchrotron spectrum in the hotspots of powerful radiogalaxies is typically observed between infrared and optical frequencies, indicating that the maximum energy of non-thermal electrons accelerated at the jet termination shock is about $1 \mathrm{TeV}$ for a canonical magnetic field of $100 \mu \mathrm{G}$. Based on theoretical considerations and observational data we show that the maximum energy of electrons cannot be constrained by synchrotron losses as usually assumed, unless the jet density is unreasonable large and most of the jet kinetic energy goes to non-thermal electrons. The maximum energy is ultimately determined by the ability to scatter particles back and forth the shock, and this limit applies to both electrons and protons. Therefore, the maximum energy of protons is also about $1 \mathrm{TeV}$ when radiative cooling is not efficient. We show that non-resonant hybrid (Bell) instabilities generated by the streaming of cosmic rays can grow fast enough to amplify the jet magnetic field up to $100 \mu \mathrm{G}$ and accelerate particles up to the maximum energies observed in the hotspots of radiogalaxies.
\end{abstract}

\section{Introduction}

Active Galactic Nuclei (AGN) host a rotating supermassive black hole surrounded by an accretion disc. Bipolar relativistic jets are launched from the inner regions of the accretion disc [1] or from the black hole ergosphere [2]. Radiogalaxies are the subclass of AGN where prominent jets are detected at radio frequencies, which in turn are classified in type I and II Faranoff-Riley (FR) galaxies [3]. Type II FR radiogalaxies exhibit well collimated jets with bright radio synchrotron knots (hotspots) at the termination region, as in Figure 1. The jet termination region is a double-shock structure composed by a bow shock in the external medium, and a reverse shock (or Mach disc) in the jet, as it is sketched in Figure 2. The velocity of the reverse shock is of the order of the jet velocity at the termination region, and therefore we will consider $v_{\text {sh }} \sim v_{\text {jet }} \sim c / 3$ [4]. Electrons radiating in the hotspot are locally accelerated in the jet reverse shock, and they reach a maximum energy $E_{e, \max }$ inferred from the Infrared (IR)/optical cut-off frequency $\left(v_{\mathrm{c}}\right)$ of the synchrotron spectrum:

$$
\frac{E_{e, \max }}{\mathrm{TeV}} \sim 0.4\left(\frac{v_{\mathrm{c}}}{10^{14} \mathrm{~Hz}}\right)^{\frac{1}{2}}\left(\frac{B}{100 \mu \mathrm{G}}\right)^{-\frac{1}{2}},
$$

where $B$ is the magnetic field [e.g. 5-9]. Ions can also be accelerated in the jet reverse shock. Given that protonproton collisions are minimal in low density plasmas such as the termination region of AGN jets, protons might achieve energies as large as the limit imposed by the size

*e-mail: $\mu$ Anabella.Araudo@eli-beams.cz of the system, usually called "Hillas limit" [10]. In particular, shocks with velocity $v_{\mathrm{sh}}=\beta_{\mathrm{sh}} c$ and Lorentz factor $\Gamma_{\mathrm{sh}}^{2}=\left(1-\beta_{\mathrm{sh}}^{2}\right)^{-1}$ might accelerate particles with Larmor radius $r_{\mathrm{g}} \sim R_{\mathrm{j}}$, where $R_{\mathrm{j}}$ is the jet radius at the termination region. Particles with such a large $r_{\mathrm{g}}$ have energy

$$
\frac{E_{\text {Hillas }}}{\mathrm{EeV}} \sim 100 \Gamma_{\mathrm{sh}}\left(\frac{\beta_{\mathrm{sh}}}{1 / 3}\right)\left(\frac{B}{100 \mu \mathrm{G}}\right)\left(\frac{R_{\mathrm{j}}}{\mathrm{kpc}}\right),
$$

as expected for Ultra High Energy Cosmic Rays (UHECRs). Type II FR radiogalaxies are located at distances larger than the Greisen-Zatsepin-Kuzmin (GZK) horizon $(\sim 100 \mathrm{Mpc})$ and therefore they cannot be the sources of the UHECRs arriving on Earth. However, to study particle acceleration in jets in FR II radiogalaxies can shed light on the ability of relativistic shocks to accelerate particles up to the Hillas limit.

The maximum energy to which particles can be accelerated via a diffusive mechanism depends on the magnetic turbulence near the shock. Theoretical results indicate that relativistic shocks are probably unable to accelerate particles to energies much larger than a PeV [12-15]. We have recently shown that the maximum energy of CR accelerated by relativistic shocks falls far short of the Hillas limit due to three main effects: 1) the steepness of the CR spectrum, 2) the small scale magnetic turbulence, and 3) the perpendicular geometry of the magnetic field $B_{0}[16]$.

In the present contribution we show that the maximum energy of protons accelerated in the hotspots of FR II radiogalaxies is significantly below $E_{\text {Hillas }}$ when the cut-off of the synchrotron spectrum is at $v_{\mathrm{c}} \sim 10^{14} \mathrm{~Hz}$ and the 


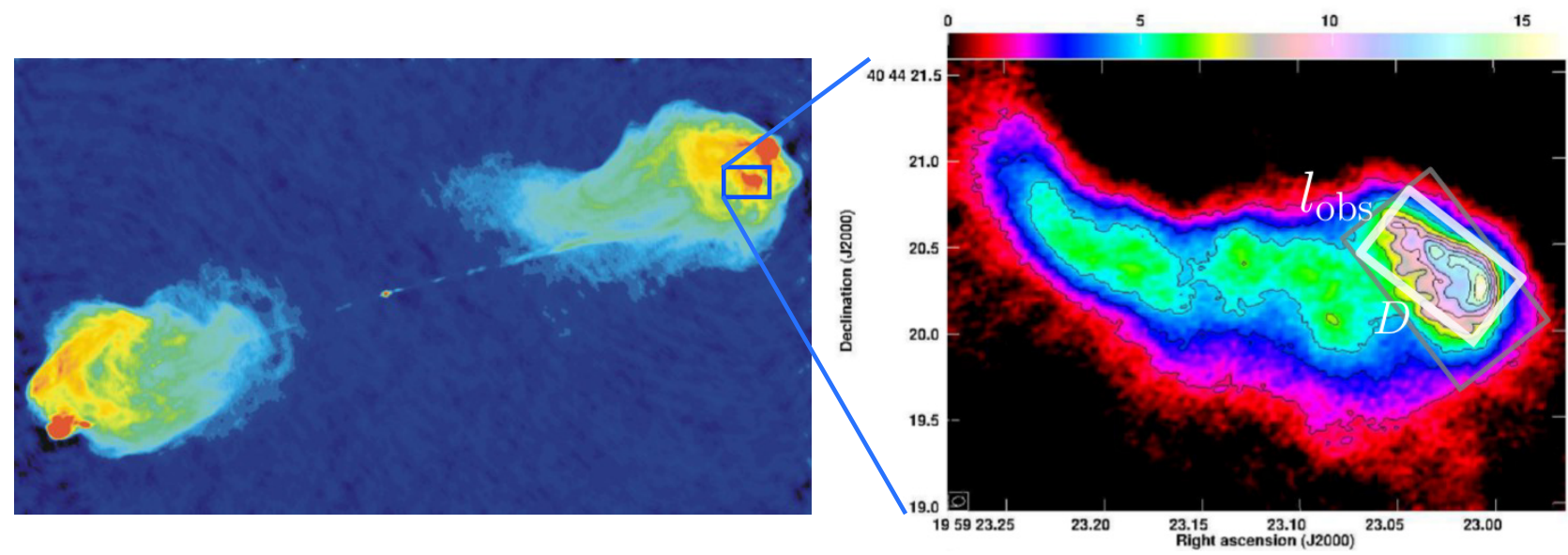

Figure 1. Left: Radiogalaxy Cygnus A at $5 \mathrm{GHz}$ (courtesy of NRAO/AUI). The jets terminate at $\sim 60 \mathrm{kpc}$ from the central source where the primary and secondary hotspots are detected. Right: Primary hotspot in the western lobe at $43 \mathrm{GHz}$, adapted from [11].

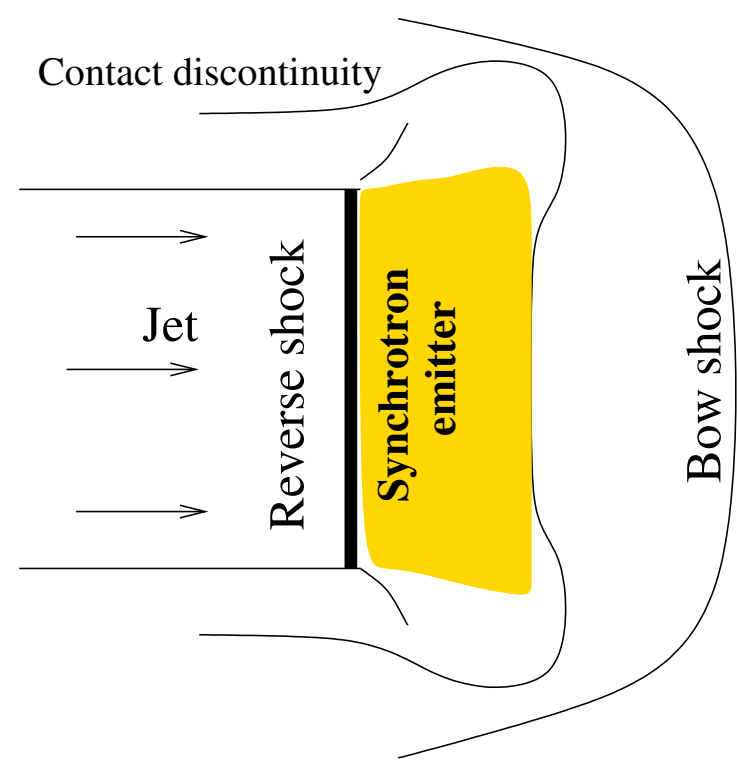

Figure 2. Sketch of the jet termination region. Particles are accelerated at the reverse shock, and radiate in the shock downstream region (the hotspot).

magnetic field in the jet reverse shock is characteristically quasi-perpendicular. In perpendicular shocks, the limit on the maximum CR energy arises from the need to amplify a turbulent magnetic field within one Larmor radius $r_{\mathrm{g} 0}$. The maximum CR energy is larger if the shock is parallel, but parallel relativistic shocks are a special and unlikely case. Throughout the paper we use cgs units and the cosmology $H_{0}=71 \mathrm{~km} \mathrm{~s}^{-1} \mathrm{Mpc}^{-1}, \Omega_{0}=1$ and $\Lambda_{0}=0.73$.

\section{On the maximum energy of electrons}

It is commonly assumed that the maximum energy of electrons accelerated in the hotspots, $E_{e, \max }$ in Eq. (1), is determined by synchrotron losses (e.g. [7]). The synchrotron cooling time of electrons with energy $E_{e, \max }$ is

$$
\frac{t_{\text {synchr }}}{\mathrm{s}} \sim 5 \times 10^{10}\left(\frac{E_{e, \mathrm{max}}}{\mathrm{TeV}}\right)^{-1}\left(\frac{B_{\mathrm{d}}}{100 \mu \mathrm{G}}\right)^{-2},
$$

where $B_{\mathrm{d}} \sim r_{B} B$ is the magnetic field in the shock downstream region, and $r_{B} \sim 3.3$ is the compression factor of the turbulent field [17]. The acceleration timescale of $E_{e, \text { max }}$-electrons in a plasma with diffusion coefficient $\mathcal{D}$ can be written as

$$
\frac{t_{\mathrm{acc}}}{\mathrm{s}} \sim 10^{3}\left(\frac{\mathcal{D}}{\mathcal{D}_{\mathrm{Bohm}}}\right)\left(\frac{E_{e, \mathrm{max}}}{\mathrm{TeV}}\right)\left(\frac{B_{\mathrm{d}}}{100 \mu \mathrm{G}}\right)^{-1}\left(\frac{v_{\mathrm{sh}}}{c / 3}\right)^{-2},
$$

where $\mathcal{D}_{\text {Bohm }}$ is the Bohm diffusion coefficient [18]. By equating $t_{\text {synchr }}=t_{\text {acc }}$, and inserting Eq. (1) in Eqs. (3) and (4), we find that

$$
\frac{\mathcal{D}}{\mathcal{D}_{\text {Bohm }}} \sim 10^{7}\left(\frac{v_{\text {sh }}}{c / 3}\right)^{2}\left(\frac{v_{\mathrm{c}}}{10^{14} \mathrm{~Hz}}\right)^{-1} .
$$

Note however that $\mathcal{D} / \mathcal{D}_{\mathrm{Bohm}}=\lambda / r_{\mathrm{g}}$, where $\lambda$ is the mean-free path and $r_{\mathrm{g}}=E_{e \text {, max }} /(e B)$ is the Larmor radius of $E_{e \text {, } \max }$-electrons in the turbulent field $B$. In the smallscale turbulence regime $\lambda=r_{\mathrm{g}}^{2} / s$, where $s$ is the plasmaturbulence scale-length [19], and therefore

$$
s \sim \frac{r_{\mathrm{g}}^{2}}{\lambda}=r_{\mathrm{g}} \frac{\mathcal{D}_{\mathrm{Bohm}}}{\mathcal{D}} .
$$

If the maximum energy of electrons is determined by synchrotron cooling, we can insert Eq. (5) in (6) resulting that

$$
s \sim 7 \times 10^{5}\left(\frac{v_{\mathrm{c}}}{10^{14} \mathrm{~Hz}}\right)^{\frac{3}{2}}\left(\frac{B}{100 \mu \mathrm{G}}\right)^{-\frac{3}{2}}\left(\frac{v_{\mathrm{sh}}}{c / 3}\right)^{-2} \mathrm{~cm} .
$$

Surprisingly, in a jet with density $n_{\text {jet }}=10^{-4} \mathrm{~cm}^{-3}$, $s$ is smaller than the ion-skin depth $c / \omega_{\mathrm{pi}} \sim$ $10^{9}\left(n_{\text {jet }} / 10^{-4} \mathrm{~cm}^{-3}\right)^{-0.5} \mathrm{~cm}$, unless $B$ is smaller than $B_{\text {max,s }}$, where

$$
\frac{B_{\mathrm{max}, \mathrm{s}}}{\mu \mathrm{G}} \sim\left(\frac{v_{\mathrm{c}}}{10^{14} \mathrm{~Hz}}\right)\left(\frac{n_{\mathrm{jet}}}{10^{-4} \mathrm{~cm}^{-3}}\right)^{\frac{1}{3}}\left(\frac{v_{\mathrm{sh}}}{c / 3}\right)^{-\frac{4}{3}} .
$$




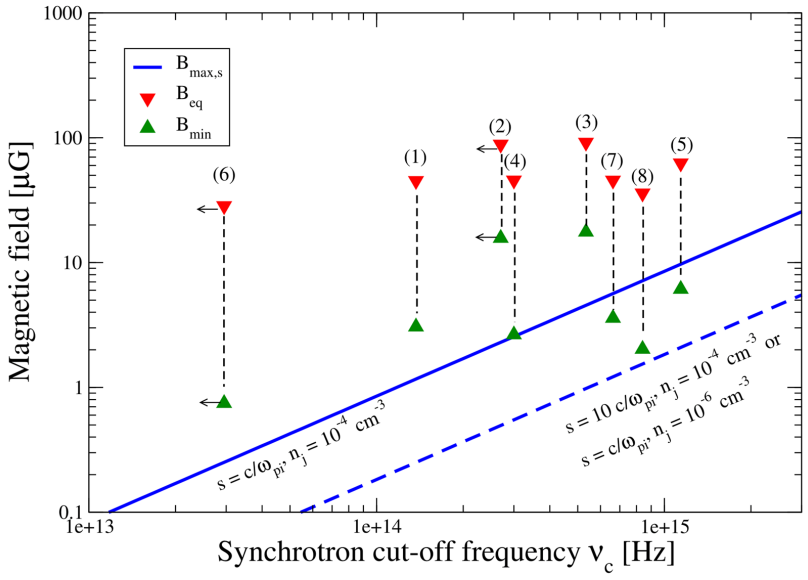

Figure 3. Upper limit $B_{\max , \mathrm{s}}$ for the magnetic field imposed by the condition $s \geq c / \omega_{p i}$ when $t_{\mathrm{acc}}=t_{\mathrm{synchr}}$. Triangles indicate the maximum $\left(B_{\text {eq }}\right.$, red triangles down) and minimum $\left(B_{\min }\right.$, green triangles up) field for the sources in [20]; see Table 1.

In Figure 3 we plot $B_{\max , \mathrm{s}}$ (blue lines) for $n_{\mathrm{jet}}=10^{-4}$ (solid line) and $10^{-6} \mathrm{~cm}^{-3}$ (dashed line). Note that the blue-dashed line represents also the case where $n_{\text {jet }}=$ $10^{-4} \mathrm{~cm}^{-3}$ and $s=10 c / \omega_{\text {pi }}$. We will see below that the small values of $B_{\max , s}$ are not enough to explain the synchrotron flux detected in hotspots.

\subsection{Synchrotron emission from hotspots}

Non-thermal electrons accelerated in the jet reverse shock are injected in the downstream region following a powerlaw energy distribution $\propto E_{e}^{-p}$, with $p=2 \alpha+1$ and being $\alpha$ the spectral index of the synchrotron radio emission. By considering the measured flux $S_{v}$ at frequency $v$ with specific luminosity $L_{v}=4 \pi d^{2} S_{v} v$ emitted in a volume $V$ at distance $d$ from Earth (see Table 1), the total energy density in relativistic electrons $U_{e}$ can be written as ${ }^{1}$

$$
\begin{aligned}
\frac{U_{e}}{\operatorname{erg~cm})^{-3}} & \sim 10^{-9}\left(\frac{p-2}{0.5}\right)^{-1}\left(\frac{v}{\mathrm{GHz}}\right)^{\frac{p-3}{2}} \\
& \left(\frac{L_{v}}{10^{41} \mathrm{erg} \mathrm{s}^{-1}}\right)\left(\frac{V}{\mathrm{kpc}^{3}}\right)^{-1}\left(\frac{B}{100 \mu \mathrm{G}}\right)^{\frac{-p-1}{2}} .
\end{aligned}
$$

The hotspot magnetic field is unknown. By setting $U_{e}(1+$ $a)=B^{2} /(8 \pi)$, where $a \geq 0$ takes into account the contribution of non-thermal protons, we find that the magnetic field in equipartition with non-thermal electrons and protons is

$$
\begin{aligned}
\frac{B_{\mathrm{eq}}}{\mu \mathrm{G}} \sim 220^{\frac{7.5}{p+5}}\left[(1+a)\left(\frac{p-2}{0.5}\right)^{-1}\right. & \\
& \left.\left(\frac{v}{8.4 \mathrm{GHz}}\right)^{\frac{p-3}{2}}\left(\frac{L_{v}}{10^{41} \mathrm{erg} \mathrm{s}^{-1}}\right)\left(\frac{V}{\mathrm{kpc}^{3}}\right)^{-1}\right]^{\frac{2}{p+5}} .
\end{aligned}
$$

On the other hand, in the extreme assumption that $U_{e}=U_{\text {kin, } \mathrm{j}}$, where $U_{\text {kin, } \mathrm{j}}=m_{p} n_{\mathrm{jet}} v_{\mathrm{sh}}^{2} / 2$ is the jet kinetic energy density, the minimum value of the magnetic field

\footnotetext{
${ }^{1}$ We assume that the minimum Lorentz factor of the electrons distribution is $\gamma_{\min }=100$ (see [21] for details).
}

required to emit a luminosity $L_{v}$ at frequency $v$ in a volume $V$ is

$$
\begin{aligned}
\frac{B_{\mathrm{min}}}{\mu \mathrm{G}} \sim 27^{\frac{3.5}{p+1}}\left(\frac{v}{\mathrm{GHz}}\right)^{\frac{p-3}{p+1}}\left(\frac{L_{v}}{10^{41} \mathrm{erg} \mathrm{s}^{-1}}\right)^{\frac{2}{p+1}} \\
{\left[\left(\frac{v_{\mathrm{j}}}{c / 3}\right)^{2}\left(\frac{p-2}{0.5}\right)\left(\frac{V}{\mathrm{kpc}^{3}}\right)\left(\frac{n_{\mathrm{jet}}}{10^{-4} \mathrm{~cm}^{-3}}\right)\right]^{\frac{-2}{p+1}} . }
\end{aligned}
$$

We compute $B_{\text {eq }}$ and $B_{\min }$ for the sample of hotspots in [20] and assuming $a=0$, as is shown in Table 1 [sources (1)-(8)] and Figure 3 . We can see that $B_{\min }>B_{\max , \mathrm{s}}$ (when $n_{\text {jet }}=10^{-4} \mathrm{~cm}^{-3}$, blue-solid line) for those sources with $v_{\mathrm{c}}<4 \times 10^{14} \mathrm{~Hz}$ whereas $B_{\min }>B_{\max , \mathrm{s}}$ in the opposite case. Note however that even when jets in radiogalaxies are expected to be perpendicular to the line of sight, a small departure from the plane of the sky reduces the size of the shock downstream region [22]. In such a case, the volume $V$ decreases and $B_{\min }$ increases whereas $B_{\max , \mathrm{s}}$ remains constant.

We note also that values of $B_{\text {min }}$ greater than those plotted in Figure 3 are expected when the jet density is smaller than $10^{-4} \mathrm{~cm}^{-3}$ (see Eq. 11). On the other hand, $B_{\max , \mathrm{s}}$ decreases when $n_{\text {jet }}<10^{-4} \mathrm{~cm}^{-3}$ (see Eq. 8). In particular, when we consider the blue-dashed line in Figure 3, sources 3C 195S, 3C 227E, 3C 445N and 3C 445S move to the regime where $B_{\min }>B_{\max , \mathrm{s}}$. The minimum value of the jet density required to match $B_{\min }=B_{\max , \mathrm{s}}$ is

$$
\begin{aligned}
\frac{n_{\text {jet,min }}}{10^{-3} \mathrm{~cm}^{-3}} \sim\left(\frac{v_{\mathrm{sh}}}{c / 3}\right)^{-\frac{4}{3}}\left(\frac{L_{v}}{10^{41} \mathrm{erg} \mathrm{s}^{-1}}\right)^{\frac{6}{p+7}}\left(\frac{v_{\mathrm{c}}}{10^{14} \mathrm{~Hz}}\right)^{\frac{3(p+1)}{p+7}} \\
{\left[\left(\frac{p-2}{0.5}\right)\left(\frac{V}{\mathrm{kpc}^{3}}\right)\right]^{\frac{-11+4 p}{p+7}}\left(\frac{v}{\mathrm{GHz}}\right)^{\frac{p-3}{p+1}}, }
\end{aligned}
$$

as listed in Table $1[21,23]$. We note however that values of $n_{\text {jet }}$ much larger than $10^{-4} \mathrm{~cm}^{-3}$ are not expected in the jet termination region. On the other hand, if the turbulence scale length is $s \sim c / \omega_{\text {pi }}$ the rapid decay of the amplified field will be in disagreement with the kpc-scale size of the synchrotron emitter.

Concerning the sources $3 \mathrm{C} 105 \mathrm{~S}$ and $3 \mathrm{C} 445 \mathrm{~S}$, both of them having large angular sizes (see Table 4 in [20]) and therefore large $V$, data with more resolution and more detailed analysis was presented in [24]. In both cases, the hotspot regions are resolved into multiple components and therefore the volume of the synchrotron emitters are smaller than the values of $V$ provided in Table 1 . In the next section we improve our calculations by removing the assumption that the jet density (at the termination region) is $n_{\text {jet }}=10^{-4} \mathrm{~cm}^{-3}$, and using also very high resolution radio data.

\section{The case study Cygnus A}

Having a redshift $z \sim 0.05607$ (i.e. a distance of $\sim 227.3 \mathrm{Mpc}$ from Earth) Cygnus A (3C 405) is the nearest FR II radiogalaxy. The primary hotspot in the western lobe in Cygnus A has been detected with very high resolution at $43 \mathrm{GHz}$, as is shown in Figure 1 [25]. Considering that the radio emitter is a cylinder of diameter 
Table 1. Physical parameters of the sources considered in this contribution. This table is adapted from [23].

\begin{tabular}{lcrc|cccr|rrrr}
\hline \hline Source & $z$ & $\begin{array}{r}v_{\mathrm{c}} \\
{\left[10^{14} \mathrm{~Hz}\right]}\end{array}$ & $\alpha$ & $p$ & $\begin{array}{c}v \\
{[\mathrm{GHz}]}\end{array}$ & $\begin{array}{r}L_{v} \\
{[\mathrm{erg} / \mathrm{s}]}\end{array}$ & $\begin{array}{r}V \\
{\left[\mathrm{kpc}^{3}\right]}\end{array}$ & $\begin{array}{r}B_{\text {eq }} \\
{[\mu \mathrm{G}]}\end{array}$ & $\begin{array}{r}B_{\min } \\
{[\mu \mathrm{G}]}\end{array}$ & $\begin{array}{r}B_{\max , \mathrm{s}} \\
{[\mu \mathrm{G}]}\end{array}$ & $\begin{array}{c}n_{\text {jet,min }} \\
{\left[\mathrm{cm}^{-3}\right]}\end{array}$ \\
\hline (1) 3C 105S & 0.089 & 1.37 & 0.75 & 2.5 & 8.4 & $1.42 \times 10^{42}$ & 1205.63 & 45.27 & 2.06 & 1.16 & $1.92 \times 10^{-4}$ \\
(2) 3C 195N & 0.110 & $<2.70$ & 0.95 & 2.9 & 8.4 & $1.15 \times 10^{41}$ & 38.12 & 75.11 & 7.89 & 2.30 & $6.51 \times 10^{-4}$ \\
(3) 3C 195S & 0.110 & 5.34 & 1.00 & 3.0 & 8.4 & $1.71 \times 10^{41}$ & 33.58 & 91.76 & 12.45 & 4.55 & $3.42 \times 10^{-4}$ \\
(4) 3C 227WE & 0.086 & 3.00 & 0.65 & 2.3 & 8.4 & $3.19 \times 10^{40}$ & 19.26 & 45.63 & 1.74 & 2.55 & $6.78 \times 10^{-5}$ \\
(5) 3C 227E & 0.086 & 11.4 & 0.75 & 2.5 & 8.4 & $7.14 \times 10^{40}$ & 17.99 & 62.60 & 4.12 & 9.71 & $3.96 \times 10^{-5}$ \\
(6) 3C 403W & 0.059 & $<0.29$ & 0.55 & 2.1 & 8.4 & $3.95 \times 10^{40}$ & 167.9 & 28.46 & 0.48 & 0.25 & $1.96 \times 10^{-4}$ \\
(7) 3C 445N & 0.056 & 6.63 & 0.85 & 2.7 & 8.4 & $2.18 \times 10^{40}$ & 29.36 & 45.60 & 2.47 & 5.65 & $3.97 \times 10^{-5}$ \\
(8) 3C 445S & 0.056 & 8.40 & 0.80 & 2.6 & 8.4 & $5.04 \times 10^{40}$ & 139.42 & 35.94 & 1.38 & 7.15 & $1.60 \times 10^{-5}$ \\
\hline (9) Cygnus A & 0.056 & $<5.00$ & 0.72 & 2.44 & 43 & $9.00 \times 10^{41}$ & 0.36 & 390 & 1.38 & 2 & $1.36 \times 10^{-4}$ \\
\hline \hline
\end{tabular}

$D=0.9$ arcsec and width (projected in the plane of the sky) $l_{\mathrm{obs}}=0.5 \mathrm{arcsec}$, the emitter volume at $43 \mathrm{GHz}$ is $V \sim 0.32 \operatorname{arcsec}^{3} \sim 0.36 \mathrm{kpc}^{3}$. Note that due to the high resolution data we are considering, the volume of the primary hotspot in Cygnus A is significantly smaller than the volume of sources (1)-(8) in Table 1 . The synchrotron luminosity at $43 \mathrm{GHz}$ is $L_{43}=9 \times 10^{41} \mathrm{erg} \mathrm{s}^{-1}$, and the spectral index from 5 to $43 \mathrm{GHz}$ is $\alpha=0.72$, and therefore $p=2 \alpha+1=2.44$ [11]. By inserting the values of $p, V, L_{v}$ and $v$ in Eqs. (10) and (11), the hotspot magnetic field needed to explain the synchrotron flux at $43 \mathrm{GHz}$ in the region indicated by the white rectangle in Figure 1 is $1.38<B / \mu \mathrm{G}<390$ when $n_{\text {jet }}=10^{-4} \mathrm{~cm}^{-3}, v_{\mathrm{sh}}=c / 3$, and $a=1$ as indicated in Table 1 . In Figure 4 we plot $B_{\min }$ and $B_{\text {eq }}$ for different values of the jet density $n_{\text {jet }}$.

The detected IR and optical emission [7, 26] indicates that the cut-off of the synchrotron spectrum is $v_{\mathrm{c}}<$ $5 \times 10^{14} \mathrm{~Hz}$. By setting $v_{\mathrm{c}}=5 \times 10^{14} \mathrm{~Hz}$, the maximum energy of non-thermal electrons accelerated at the jet reverse shock is $E_{e, \max } \sim 0.8 \mathrm{TeV}$ when $B=100 \mu \mathrm{G}$. In Figure 4 we plot $B_{\max , \mathrm{s}}$ as a function of the jet density. We can see that $B_{\min }>B_{\max , \mathrm{s}}$ for all possible values of $n_{\text {jet }}$, and therefore $E_{e, \max }$ cannot be determined by synchrotron cooling in the primary hotspot of Cygnus A, in disagreement with the standard assumption as was pointed out by [27] and [28].

\section{On the maximum energy of particles in perpendicular shocks}

If synchrotron losses do not balance energy gain, the electrons' maximum energy $E_{e \text {, max }}$ is ultimately determined by the ability to scatter particles in the shock environment, and this limit applies to both electrons and protons. Therefore, the maximum energy of protons, $E_{p \text {, max }}$, is the same as the electrons' maximum energy and we will call it $E_{\max }=E_{p, \max }=E_{e, \max }$. Relativistic shocks are characteristically quasi-perpendicular, i.e. $\mathbf{B}_{\mathbf{0}} \perp \mathbf{v}_{\mathrm{sh}}$, where $B_{0}$ is the (unperturbed) jet magnetic field at the termination region. To accelerate particles up to an energy $E_{\max }$ in perpendicular shocks, the mean-free path in turbulent magnetic field in the shock downstream region, $\lambda_{\mathrm{d}} \sim\left(E_{\max } / e B\right)^{2} / s$ has to be smaller than Larmor radius $r_{\mathrm{g} 0}$ in the ordered (and compressed) field $B_{\mathrm{jd}} \sim 4 B_{0}$ in order to avoid the particles following the $B_{\mathrm{jd}}$-helical orbits and cross-field diffu-

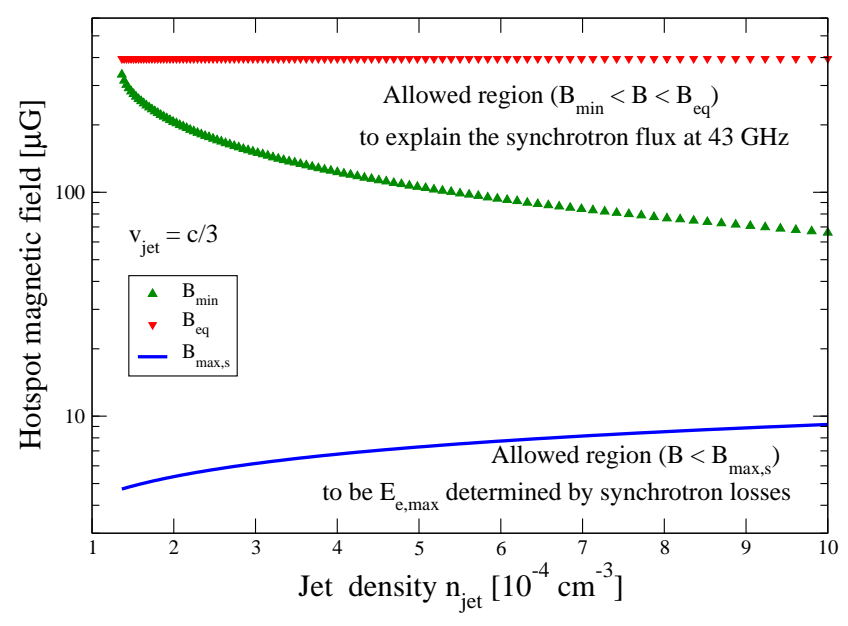

Figure 4. Comparison between the magnetic field required to explain the synchrotron flux at $43 \mathrm{GHz}\left(B_{\min } \leq B \leq B_{\text {eq }}\right)$ and the magnetic field required to satisfy the condition $s \leq c / \omega_{\text {pi }}$ $\left(B \leq B_{\max , \mathrm{s}}\right)$

sion ceasing [e.g. 14, 15, 29]. The condition $\lambda_{\mathrm{d}}<r_{\mathrm{g} 0}$ is marginally satisfied when the magnetic-turbulence scalelength is $s=s_{\perp}$, where

$$
s_{\perp}=\frac{E_{\max }}{e B}\left(\frac{4 B_{\mathrm{j}}}{B}\right) .
$$

By inserting Eq. (1) in Eq. (13) we find that

$$
s_{\perp} \sim 3 \times 10^{11}\left(\frac{v_{\mathrm{c}}}{10^{14} \mathrm{~Hz}}\right)^{\frac{1}{2}}\left(\frac{B_{0}}{\mu \mathrm{G}}\right)\left(\frac{B}{100 \mu \mathrm{G}}\right)^{-\frac{5}{2}} \mathrm{~cm} .
$$

By considering the values of $B_{\text {eq }}$ and $B_{\min }$ in Figure 4, and fixing $B_{0}=1 \mu \mathrm{G}$ and $v_{\mathrm{c}}=5 \times 10^{14} \mathrm{~Hz}$, we plot $s_{\perp}$ in Figure 5 for the primary hotspot of Cygnus A. Note that $s_{\perp}>c / \omega_{\mathrm{pi}}$ indicating that the magnetic field is not generated by the Weibel instability (that has a characteristic scale length of $\left.c / \omega_{\mathrm{pi}}\right)$.

\section{Bell instabilities in perpendicular shocks}

Turbulence on a scale greater than $c / \omega_{\text {pi }}$ may be excited through the Bell instability, which can grow until 


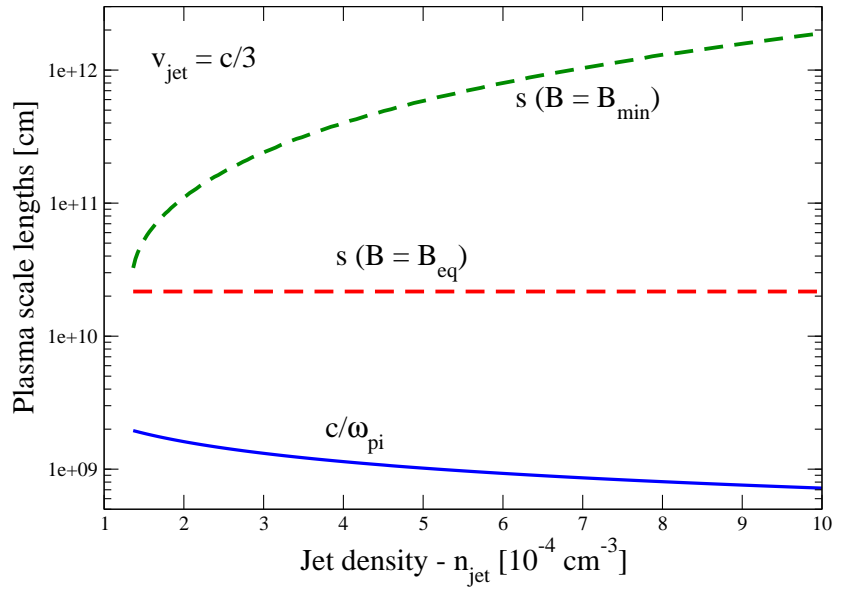

Figure 5. Magnetic turbulence scale length $s_{\perp}$ (dashed lines) and ion-skin-depth $c / \omega_{\mathrm{pi}}$ in the primary hotspot in Cygnus A. (See [28] for the same plot with different values of $v_{\text {jet }}$.)

$s_{\perp}$ reaches the Larmor radius of the highest energy CR driving the instability $[30,31]$. In perpendicular shocks, the Bell instability growth rate is smaller by a numerical factor of the order of one than in the parallel case [31] (see also [32, 33]), and therefore $\Gamma_{\max } \sim 0.5 j_{\mathrm{cr}} \sqrt{4 \pi / \rho_{\text {jet, }} \text {, }}$ where $\rho_{\text {jet,d }}=4 m_{p} n_{\text {jet }}$ is the density in the shock downstream region and $j_{\mathrm{cr}}$ is the CR current, is a good order-ofmagnitude measure of the growth rate. Because we are interested on the turbulence generated by the highest energy CR with energy $E_{\max }$, the current driven by these particles is $j_{\mathrm{cr}} \sim U_{p} e v_{\mathrm{sh}} / E_{\mathrm{max}}$, where the protons energy density can be written as $U_{p} \sim \eta U_{\mathrm{kin}, \mathrm{j}}$, and $\eta$ is the CR acceleration efficiency.

The condition for magnetic field amplification by the Bell instability in perpendicular shocks is $\Gamma_{\max } t_{\perp}>10$, where $t_{\perp}=4 r_{\mathrm{g} 0} / v_{\mathrm{sh}}$ is the time during which the plasma flows through a distance $r_{\mathrm{g} 0}$ in the downstream region at velocity $v_{\mathrm{sh}} / 4$. This condition leads to a lower limit on the $\mathrm{CR}$ acceleration efficiency

$$
\eta>\eta_{\min }=0.057\left(\frac{v_{\mathrm{sh}}}{c / 3}\right)^{-1}\left(\frac{B_{0}}{\mu \mathrm{G}}\right)\left(\frac{n_{\mathrm{jet}}}{10^{-4} \mathrm{~cm}^{-3}}\right)^{-\frac{1}{2}} .
$$

In order to check whether this condition is satisfied in the hotspots, we consider that CRs are accelerated in the jet reverse shock following a power-law energy distribution with the same index $p$ as non-thermal electrons. Therefore, the $E_{\max }-\mathrm{CR}$ acceleration efficiency is

$$
\eta_{E_{\max }} \sim(p-2)\left(\frac{U_{\mathrm{cr}}}{U_{\mathrm{kin}, \mathrm{j}}}\right)\left(\frac{E_{\max }}{\mathrm{GeV}}\right)^{2-p},
$$

where the total energy density in CRs is $U_{\mathrm{cr}}=U_{e}$ (Eq. 9), as expected in relativistic shocks (i.e. $a=1$ ). Therefore, to satisfy the condition $\eta_{E_{\max }}>\eta_{\min }$ (Eq. 15) the jet (unperturbed) magnetic field has to be

$$
\begin{aligned}
\left(\frac{B_{0}}{\mu \mathrm{G}}\right) & <2\left(\frac{n_{\text {jet }}}{10^{-4} \mathrm{~cm}^{-3}}\right)^{-\frac{1}{2}}\left(\frac{B}{100 \mu \mathrm{G}}\right)^{-1}\left(\frac{L_{v}}{10^{41} \mathrm{erg} \mathrm{s}^{-1}}\right) \\
& \left(\frac{v_{\mathrm{c}}}{10^{14} \mathrm{~Hz}}\right)^{\frac{2-p}{2}}\left(\frac{v}{\mathrm{GHz}}\right)^{\frac{p-3}{2}}\left(\frac{V}{\mathrm{kpc}^{3}}\right)^{-1} .
\end{aligned}
$$

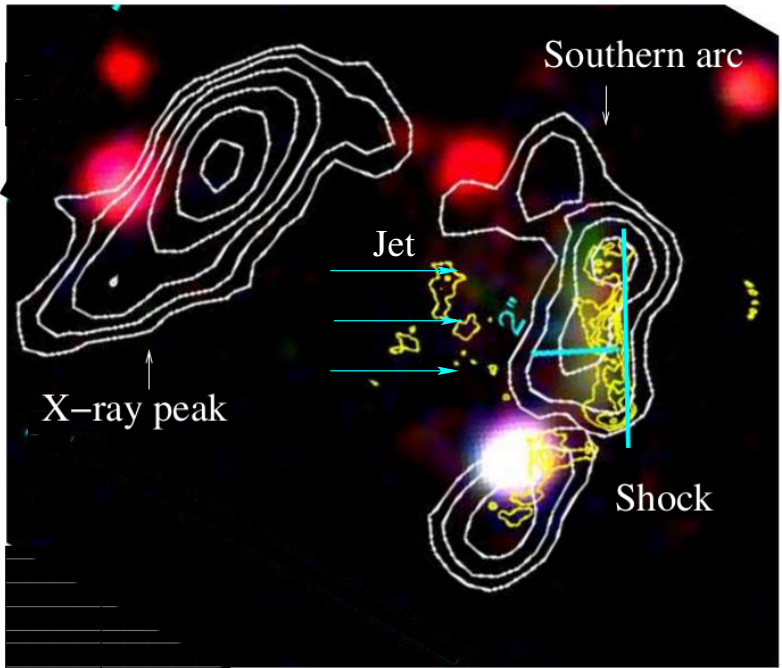

Figure 6. Hotspot at the southern jet of the FRII galaxy 4C74.26. White and yellow contours are X-rays and (MERLIN) radio data, respectively. Red and green correspond to IR and optical, respectively. One arcsecond represents $1.887 \mathrm{kpc}$ on the plane of the sky at the location of the source $(z=0.104)$. Adapted from [35].

In such a case, CRs with energy $E_{\max }$ have sufficient energy density to generate non-resonant turbulence on scale $s_{\perp}$ in the hotspots of FRII radiogalaxies. In the particular case of the primary hotspot in Cygnus A, the condition in Eq. (17) reduces to

$$
\left(\frac{B_{0}}{\mu \mathrm{G}}\right)<0.24\left(\frac{n_{\text {jet }}}{10^{-4} \mathrm{~cm}^{-3}}\right)^{-\frac{1}{2}}\left(\frac{B}{100 \mu \mathrm{G}}\right)^{-1.28} .
$$

We note that evidence of magnetic field amplification and damping was found in the jet termination region of the quasar 4C74.26 [34]. The compact synchrotron emission $(\sim 0.1 \mathrm{kpc})$ detected in the southern hotspot of this source would require a magnetic field $\sim 2.4 \mathrm{mG}$ to match the size of the emitter with the synchrotron cooling length at the observed frequency of $1.66 \mathrm{GHz}$ (see Figure 6). This value of the magnetic field is about 10 times the upper limit imposed by the equipartition condition with non-thermal particles (see more details of the model in [34]). Therefore, the compact radio emission delineates the region within which the magnetic field is amplified by plasma instabilities up to $\sim 100 \mu \mathrm{G}$ and it is damped downstream of the shock.

\section{Conclusions}

We have studied the physical mechanism that constraints the maximum energy of particles accelerated at the hotspots of FRII radiogalaxies. Based on one observable (the cut-off $v_{\mathrm{c}}$ of the synchrotron spectrum) and one (17) physical requirement $\left(s \geq c / \omega_{p i}\right)$ we have found that extreme conditions in the jet plasma would be required for $E_{e \text {, max }} \sim 1 \mathrm{TeV}$ to be determined by synchrotron cooling, 
as usually assumed. By equating the acceleration and synchrotron cooling timescales, the mean free path of $E_{e, \max ^{-}}$ electrons is greater than the maximum value imposed by plasma physics for reasonable values of the magnetic field and jet density. By considering a sample of hotspots we show that unreasonably large values of the jet density would be required to explain the radio synchrotron flux is determined by synchrotron cooling (see Eq.12).

The maximum energy $E_{e \text {, max }}$ is ultimately determined by the scattering process. By assuming that the shock is quasi-perpendicular, particles cannot diffuse further than a distance $r_{\mathrm{g} 0}$ downstream of the shock. To satisfy the condition $\lambda_{\mathrm{d}}<r_{\mathrm{g} 0}$, the magnetic turbulence scale-length has to be $s_{\perp} \sim 2 \times 10^{12} \mathrm{~cm}$, that is $\sim 1000 \mathrm{c} / \omega_{\mathrm{pi}}$, and therefore $B$ is not amplified by the Weibel turbulence. On the other hand, the Bell instability amplify the magnetic field on scales larger than $c / \omega_{\text {pi }}$ and we show that Bell-modes generated by CRs with energies $E_{e \text {,max }}$ can grow fast enough to accelerate particles up to maximum energies $\sim 1 \mathrm{TeV}$ observed in the hotspots of FRII radiogalaxies. The advantage of magnetic turbulence being generated by CRs current is that $B$ persists over long distances downstream of the shock, and therefore particles accelerated very near the shock can emit synchrotron radiation far downstream.

Finally, if $E_{e, \max }$ is determined by the diffusion condition in a perpendicular shock, the same limit applies to protons and therefore the maximum energy of ions is also $\sim 1 \mathrm{TeV}$. As a consequence, relativistic shocks in the termination region of FR II jets are poor cosmic ray accelerators. Motivated by this result, we have recently performed hydrodynamic simulations and shown that mildly relativistic shocks in the backflowing material of radio galaxy lobes can accelerate UHECRs [36]. These shocks occur after the jet material has passed through the relativistic termination shock where the magnetic field is amplified.

\section{Knowledgments}

We thank the organisers of UHECR 2018 for an excellent conference. This work is supported by the Science and Technology Facilities Council under consolidated grant ST/N000919/1. A.T.A. thanks the Czech Science Foundation (ref. 14-37086G) - "Albert Einstein Center for Gravitation and Astrophysics" in Prague, and the EU COST Action (ref. CA16104) "Gravitational waves, black holes and fundamental physics"

\section{References}

[1] R.D. Blandford, D.G. Payne, 199, 883 (1982)

[2] R.D. Blandford, R.L. Znajek, 179, 433 (1977)

[3] B.L. Fanaroff, J.M. Riley, 167, 31P (1974)

[4] F. Casse, A. Marcowith, Astroparticle Physics 23, 31 (2005), astro-ph/0411395

[5] K. Meisenheimer, M.G. Yates, H.J. Roeser, 325, 57 (1997)

[6] G. Brunetti, K.H. Mack, M.A. Prieto, S. Varano, 345, L40 (2003), astro-ph/0309290
[7] Ł. Stawarz, C.C. Cheung, D.E. Harris, M. Ostrowski, 662, 213 (2007), astro-ph/0701568

[8] J. Zhang, J.M. Bai, L. Chen, E. Liang, 710, 1017 (2010), 0912.2470

[9] M.W. Werner, D.W. Murphy, J.H. Livingston, V. Gorjian, D.L. Jones, D.L. Meier, C.R. Lawrence, 759, 86 (2012), 1209.0810

[10] A.M. Hillas, 22, 425 (1984)

[11] S. Pyrzas, K.C. Steenbrugge, K.M. Blundell, 574, A30 (2015)

[12] J.G. Kirk, B. Reville, 710, L16 (2010), 1001.0687

[13] M. Lemoine, G. Pelletier, 402, 321 (2010), 0904.2657

[14] L. Sironi, A. Spitkovsky, J. Arons, 771, 54 (2013), 1301.5333

[15] B. Reville, A.R. Bell, 439, 2050 (2014), 1401. 2803

[16] A.R. Bell, A.T. Araudo, J.H. Matthews, K.M. Blundell, 473, 2364 (2018), 1709.07793

[17] E. Parizot, A. Marcowith, J. Ballet, Y.A. Gallant, 453, 387 (2006), astro-ph/0603723

[18] P.O. Lagage, C.J. Cesarsky, 125, 249 (1983)

[19] M. Ostrowski, J. Bednarz, 394, 1141 (2002), astro-ph/0101069

[20] K.H. Mack, M.A. Prieto, G. Brunetti, M. Orienti, 392, 705 (2009), 0810. 3764

[21] M. Orienti, M.A. Prieto, G. Brunetti, K.H. Mack, F. Massaro, D.E. Harris, 419, 2338 (2012), 1109.4895

[22] K. Meisenheimer, H.J. Roser, P.R. Hiltner, M.G. Yates, M.S. Longair, R. Chini, R.A. Perley, 219, 63 (1989)

[23] A.T. Araudo, Boletin de la Asociacion Argentina de Astronomia La Plata Argentina 60, 79 (2018)

[24] M. Orienti, M.A. Prieto, G. Brunetti, K.H. Mack, F. Massaro, D.E. Harris, 419, 2338 (2012), 1109.4895

[25] S. Pyrzas, K.C. Steenbrugge, K.M. Blundell, 574, A30 (2015)

[26] K. Nilsson, M.J. Valtonen, L.R. Jones, W.C. Saslaw, H.J. Lehto, 324, 888 (1997)

[27] A.T. Araudo, A.R. Bell, A. Crilly, K.M. Blundell, 460, 3554 (2016), 1605.05125

[28] A.T. Araudo, A.R. Bell, K.M. Blundell, J.H. Matthews, 473, 3500 (2018), 1709.09231

[29] J.G. Kirk, B. Reville, 710, L16 (2010), 1001. 0687

[30] A.R. Bell, 353, 550 (2004)

[31] A.R. Bell, 358, 181 (2005)

[32] M.A. Riquelme, A. Spitkovsky, 717, 1054 (2010), 0912. 4990

[33] J.H. Matthews, A.R. Bell, K.M. Blundell, A.T. Araudo, 469, 1849 (2017), 1704.02985

[34] A.T. Araudo, A.R. Bell, K.M. Blundell, 806, 243 (2015), 1505.02210

[35] M.C. Erlund, A.C. Fabian, K.M. Blundell, C.S. Crawford, P. Hirst, 404, 629 (2010), 1001.1063

[36] J.H. Matthews, A.R. Bell, K.M. Blundell, A.T. Araudo, 482, 4303 (2019), 1810. 12350 